\title{
Progress in constraining the asymmetry dependence of the nuclear caloric curve
}

\author{
Alan B. McIntosh and Sherry J. Yennello
}

Cyclotron Institute and Department of Chemistry, Texas A\&M University, College Station TX, USA

\begin{abstract}
The nuclear equation of state is a basic emergent property of nuclear material. Despite its importance in nuclear physics and astrophysics, aspects of it are still poorly constrained. Our research focuses on answering the question: How does the nuclear caloric curve depend on the neutron-proton asymmetry? We briefly describe our initial observation that increasing neutron-richness leads to lower temperatures. We then discuss the status of our recently executed experiment to independently measure the asymmetry dependence of the caloric curve.
\end{abstract}

The nuclear equation of state (EOS) relates the thermodynamic state variables temperature, pressure, density, internal energy, and neutronproton asymmetry. It describes the evolution of finite excited nuclear systems created in terrestrial accelerator experiments and finds application in many aspects of nuclear astrophysics as well. Our knowledge of the EOS is well constrained for nuclear systems near beta stability. However, extrapolation to very exotic environments far from beta stability (e.g. neutron stars) represents the largest uncertainty in the EOS. We approach this deficiency in a unique way by seeking to answer the question: How does the caloric curve depend on the neutron-proton asymmetry?

To date, the theoretical approaches [1-6] that have been applied to this question have provided answers that differ not only in magnitude but in sign: some predict a decrease in temperature with increasing neutron content, while others predict just the opposite. This discrepancy stems in part 


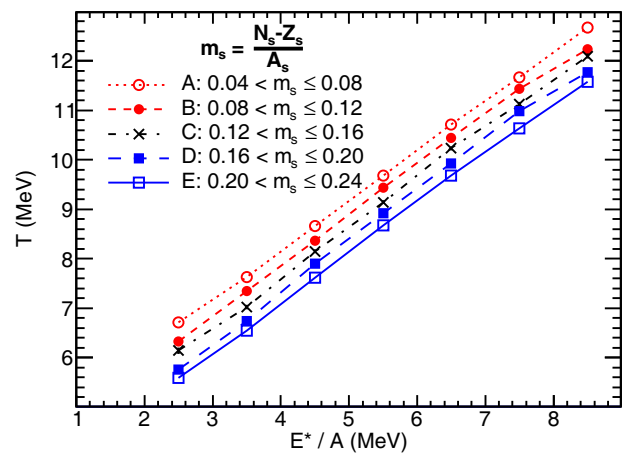

Figure 1: For reconstructed, isotopically-resolved quasi-projectile sources, the caloric curve shifts to lower temperature with increasing asymmetry.

from a dearth of experimental constraints. This paper summarizes our first observation of dependence to the caloric curve, and describes the status of our subsequent measurement for which the analysis is in progress. We hope that these measurements may stimulate and guide theoretical progress.

We have previously reported our observation of the asymmetry dependence of the nuclear caloric curve [7-9]. Charged particles and neutrons produced in reactions of ${ }^{70} \mathrm{Zn}+{ }^{70} \mathrm{Zn},{ }^{64} \mathrm{Zn}+{ }^{64} \mathrm{Zn},{ }^{64} \mathrm{Ni}+{ }^{64} \mathrm{Ni} @ 35 \mathrm{~A} \mathrm{MeV}$ were measured with the $4 \pi$ NIMROD-ISiS array at Texas A\&M University Cyclotron Institute. Charged particles were isotopically identified using the $\Delta \mathrm{E}$-E technique up to at least $\mathrm{Z}=17$ in many detectors. We recognized that it was crucial to reconstruct the quasi-projectile (QP), the primary excited large piece of nuclear material produced near beam velocity in non-central collisions. Such a reconstruction allowed us to select on the neutron-proton asymmetry of the QP rather than the asymmetry of the entrance channel. This provides a much more sensitive measurement of the asymmetry dependence of the caloric curve. Building on the method of Wuenschel et al. [10], we selected events in which the charged particles comprising the QP were all isotopically identified and further required that the reconstructed QP be thermally equilibrated by selecting events which are on average spherically symmetric. Excitation energies were calculated from measured kinetic energies of charged fragments, the neutron multiplicity and average kinetic energy, and the Q-value of the QP breakup.

Temperatures were calculated using three methods: the momentum quadrupole fluctuation thermometer (MQF) [11], the Albergo yield ratio [12], and the slope-temperature method [13]. The first and third of these both make use of the momenta of charged particles, while the second is a 


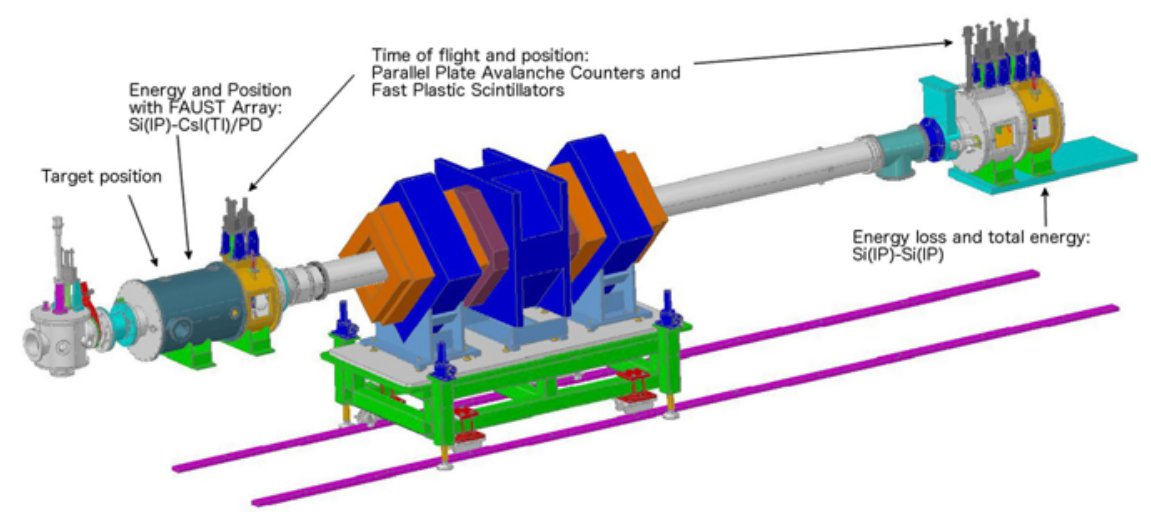

Figure 2: The quadrupole triplet spectrometer at TAMU provides a time-of-flight measurement for heavy ions around zero degrees $\left(0.9^{\circ} \leq \theta_{l a b} \leq 2.3^{\circ}\right)$. The FAUST array complements the QTS by providing charged particle measurement over a large angular range.

chemical method. The caloric curve, the correlation between the temperature and excitation energy of the reconstructed QP, is shown in Fig. 1 using the MQF method with protons as the probe particle. As expected, the temperature rises with increasing excitation energy. Due to the relatively light mass of the QP $\left(48 \leq A_{s} \leq 52\right)$, the plateau is expected to begin around $E * / A \approx 9 \mathrm{MeV}$ [14], which is above the energies probed here. The data is broken into 5 ranges of asymmetry $\left(m_{s}=\frac{N_{s}-Z_{s}}{A_{s}}\right)$ which span a broad range in asymmetry. For any given excitation energy, as the source becomes more neutron-rich, the temperature decreases. This occurs relatively uniformly for all excitation energies measured. A change from $m_{s}=0.06$ to 0.22 for this $A \approx 50$ system corresponds to 4 neutrons, and elicits a decrease in temperature of $1.1 \mathrm{MeV}$. Other charged particle probes of the temperature using all methods mentioned above provide the same result that a larger neutron content corresponds to a decrease in the temperature. The magnitude of the effect differs from one probe to another (between 0.1 and $2.2 \mathrm{MeV}$ ), and in all cases is statistically significant. In addition to verifying this initial observation, future work should attempt to constrain the magnitude of the effect.

We have designed and executed an experiment to independently measure the asymmetry dependence of the caloric curve by studying incomplete fusion reactions of ${ }^{78,86} \mathrm{Kr}+{ }^{12} \mathrm{C} @ 15,25,35 \mathrm{~A} \mathrm{MeV}$. The excitation and composition of the compound nuclei produced are determined by the fraction of the target that fuses with the projectile and does not rely on a free 


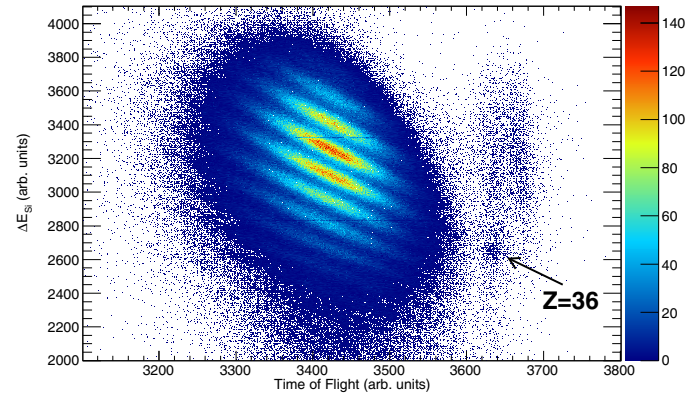

Figure 3: Particles can be identified by atomic number in the QTS by the correlation between energy loss and time of flight. Data shown is measured within $0.9^{\circ}<\theta_{l a b}<$ $2.3^{\circ}$ for ${ }^{86} \mathrm{Kr}+{ }^{12} \mathrm{C} @ 25 \mathrm{~A} \mathrm{MeV}$.

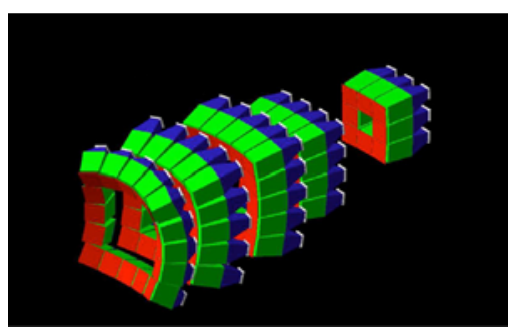

Figure 4: The FAUST array provides nearly complete coverage for charged particles in the angular range $2.3^{\circ}<\theta_{\text {lab }}<34.1^{\circ}$ with partial coverage for $\theta_{\text {lab }}<45.5$ and $\theta_{l a b}>1.6$.

neutron measurement. The fraction of the targets that fuses with the projectile can be deduced by measuring the velocity of the fusion-evaporation residue event-by-event [15], and applying a correction $(<10 \%)$ for the recoil of the residual target-like fragment [16]. The temperature can be obtained by measuring charged particles evaporated from the compound nucleus and then employing the methods discussed above.

The experiment was conducted using the FAUST-QTS detector system at the Texas A\&M University Cyclotron Institute in July-August 2015. Fig. 2 illustrates shows a mechanical drawing of the experimental apparatus. The krypton beams entered from the left and collided with an $830 \mu \mathrm{g}$ carbon target. Light charged particles were measured in the FAUST array (discussed in more detail below). Downstream of FAUST, a Faraday cup collected unreacted and elastically-scattered beam with $\theta_{l a b}<0.9^{\circ}$. Fragments in the angular range $0.9^{\circ}<\theta_{l a b}<2.3^{\circ}$ were focused by the quadrupole triplet spectrometer (QTS), over $\mathrm{a} \approx 5.5 \mathrm{~m}$ flight path. On either end of this 


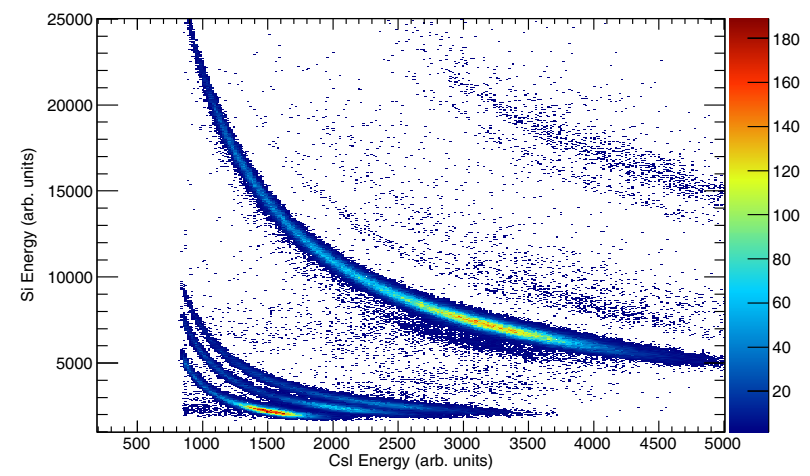

Figure 5: Light charged particles are identified in FAUST using the dE-E technique. Data shown is for ${ }^{86} \mathrm{Kr}+{ }^{12} \mathrm{C} @ 25 \mathrm{~A} \mathrm{MeV}$, FAUST telescope 49.

flight path, parallel plate avalanche detectors and thin plastic scintillator detectors were used to measure the time of flight. At the downstream end, silicon detectors were used to measure energy loss and total energy. Figure 3 shows the correlation between the energy loss and the time of flight for reactions of ${ }^{86} \mathrm{Kr}+{ }^{12} \mathrm{C} @ 25 \mathrm{~A} \mathrm{MeV}$. With the timing resolution in the vicinity of 500ps and energy on the $1 \%$ level, determination of the atomic number of the heavy ion is possible with unit resolution. A small contribution of elastically scattered beam can be used to identify the $Z=36$ band. Further details of the FAUST-QTS detector system may be found in references $[17,18]$.

Figure 4 shows a GEANT4 model of the $68 \mathrm{Si}-\mathrm{CsI}(\mathrm{Tl}) / \mathrm{PD}$ detector telescopes of the FAUST array. These provide $\approx 95 \%$ coverage in the angular range $2.3^{\circ}<\theta_{l a b}<34.1^{\circ}$ with partial coverage for $\theta_{\text {lab }}>1.6^{\circ}$ and $\theta_{\text {lab }}<45.5^{\circ}$. According to the evaporation codes GEMINI++ and PACE4, this range provides kinematic coverage for the vast majority of the phase space for light charged particles in the reactions studied. The silicon detectors are position sensitive to better than $200 \mu m$ [19]. Particle Identification in FAUST uses the $\Delta \mathrm{E}-\mathrm{E}$ technique. Figure 5 shows the isotopic resolution obtained for isotopes of hydrogen and helium obtained for detector 49 in reactions of ${ }^{86} \mathrm{Kr}+{ }^{12} \mathrm{C} @ 25 \mathrm{~A} \mathrm{MeV}$. Details of the FAUST array may be found in references [19-21].

With the excellent coverage and resolution of the evaporated particles and the fusion-evaporation residues in these measurements of $\mathrm{Kr}+\mathrm{C}$, we expect to provide constraints on the dependence of the nuclear caloric curve on the neutron-proton asymmetry. 


\section{Acknowledgement}

This work was supported by the Robert A. Welch Foundation (Grant A-1266) and the United States Department of Energy (Grant DE-FG0293ER40773).

\section{References}

[1] J. Besprosvany, S. Levit, Phys. Lett. B 217, 1 (1989)

[2] Y.J. Zhang et al., Phys. Rev. C 54, 1137 (1996)

[3] V.M. Kolomietz, A.I. Sanzhur, S. Shlomo, S.A. Firin, Phys. Rev. C 64, 024315 (2001)

[4] R. Ogul, A.S. Botvina, Phys. Rev. C 66, 051601(R) (2002)

[5] C. Hoel, L.G. Sobotka, R.J. Charity, Phys. Rev. C 75, 017601 (2007)

[6] J. Su, F.S. Zhang, Phys. Rev. C 84, 037601 (2011)

[7] A.B. McIntosh et al., Phys. Lett. B 719, 337 (2013)

[8] A.B. McIntosh et al., Phys. Rev. C 87, 034617 (2013)

[9] A.B. McIntosh et al., Euro. Phys. J A 50, 35 (2014)

[10] S. Wuenschel et al., Phys. Rev. C 79, 061602(R) (2009)

[11] H. Zheng, A. Bonasera, Phys. Lett. B 696, 178 (2011)

[12] S. Albergo, S. Costa, E. Costanzo, A. Rubbino, Il Nuovo Cimento 89, 1 (1985)

[13] R. Yanez et al., Phys. Rev. C 68, 011602(R) (2003)

[14] J.B. Natowitz et al., Phys. Rev. C 65, 034618 (2002)

[15] H. Morgenstern et al., Phys. Lett. B 113, 463 (1982)

[16] W. Bohne et al., Rev. Mod. Phys. 41, 5 (1990)

[17] P. Cammarata et al., Nucl. Inst. Meth. A 792, 61 (2015)

[18] A.B. McIntosh et al., Nucl. Inst. Meth. A (2016), to be submitted 
[19] S. Soisson et al., Nucl. Inst. Meth. A 613, 240 (2010)

[20] F. Gimeno-Nogues et al., Nucl. Inst. Meth. A 399, 94 (1997)

[21] L. Heilborn et al., Nucl. Inst. Meth. A (2016) 